\title{
Carrières migratoires des femmes latino- américaines dans le secteur de la domesticité à Bruxelles
}

Migratory Careers of Latin-American Women in the Domestic Sector in Brussels Carreras migratorias de las mujeres latinoamericanas en el sector de la domesticidad de Bruselas

Any Freitas et Marie Godin

\section{(2) OpenEdition}

Édition électronique

URL : https://journals.openedition.org/remi/6381

DOI : 10.4000/remi.6381

ISSN : $1777-5418$

Éditeur

Université de Poitiers

Édition imprimée

Date de publication : 1 juin 2013

Pagination : 37-55

ISBN : 979-10-90426-08-5

ISSN : 0765-0752

Référence électronique

Any Freitas et Marie Godin, « Carrières migratoires des femmes latino-américaines dans le secteur de la domesticité à Bruxelles », Revue européenne des migrations internationales [En ligne], vol. 29 - n² | 2013, mis en ligne le 01 juin 2016, consulté le 15 avril 2022. URL : http://journals.openedition.org/ remi/6381; DOI : https://doi.org/10.4000/remi.6381 


\title{
Carrières migratoires des femmes latino-américaines dans le secteur de la domesticité à Bruxelles
}

\author{
Any Freitas ${ }^{1}$ et Marie Godin ${ }^{2}$
}

\section{Introduction}

Cet article analyse l'évolution des "carrières migratoires " (Martiniello et Rea, 2011) des femmes migrantes latino-américaines, insérées dans le secteur de la domesticité (ou, plus généralement, dans le care) à Bruxelles. Souvent en situation irrégulière, elles sont venues de différents pays de la région, suite aux crises économiques qui s'y sont succédé entre les années 1980 et le début des années 2000. La demande grandissante de main-d'œuvre, dans une ville que I'on peut qualifier de "global-city Region" (Favell, 2009), a renforcé la position des Latino-américaines dans cette niche économique. La structuration du secteur du marché de la domesticité informelle ne peut cependant se comprendre en dehors des dynamiques plus globales des migrations internationales. Comme le montre nombre de travaux (voir Hochschild, 2000 ; Lutz, 2008), il existe une forte interdépendance entre la migration des travailleurs qualifiés et celles des travailleurs non qualifiés. Selon Portes et al. (1999), on assisterait à une mondialisation par le haut des élites et à une mondialisation par le bas des migrants pour la gestion des activités que les familles externalisent.

Afin d'explorer l'immigration féminine latino-américaine, très souvent considérée, à tort, comme un groupe homogène, nous avons identifié deux groupes à l'intérieur de la catégorie ; le premier rassemble des femmes équatoriennes, colombiennes et boliviennes, et le second, celles qui viennent du Brésil. Bien qu'elles présentent de nombreux points communs, l'évolution des carrières migratoires des femmes de ces deux groupes n'en reste pas moins contrastée à bien des égards. En effet, même si elle tend à se ressembler du point de vue de l'analyse des niches de travail, des lieux d'installation et des espaces de socialisation, la diversité des origines continue à être une variable importante pour rendre compte notamment de la différence des trajectoires des migrantes latino-américaine.

\footnotetext{
1 Chargée de Programme Senior, Institut d'études de sécurité de I'Union européenne, 100 avenue de Suffren, 75007 Paris ; any.freitas@eui.eu

2 Chercheuse așsociée au Groupe de recherche sur les Relations Ethniques, les Migrations et I'Égalité (GERME, Université Libre de Bruxelles) et PhD Student University of East London (UK), 11 Rum Close, E1W 30X, London ; godinmarie@gmail.com
} 
À partir d'un travail de terrain réalisé à Bruxelles entre juin 2009 et mars 2010, cet article propose une analyse des différentes " portes d'entrée " pour trouver une place sur le marché de la domesticité. L'approche est fondée sur le concept de " carrière migratoire " qui permet de s'écarter du cadre habituel fréquemment rencontré dans la littérature sur la domesticité, lequel décrit bien souvent de manière indépendante les sphères dites du "live-in " versus "live-out ". II offre la possibilité de conjuguer les dimensions objectives (parcours juridicoinstitutionnel et socio-économique) et subjectives (évolution des identités, projets et statuts) dans l'analyse des carrières des femmes migrantes et de saisir la façon dont les différentes conceptions " de soi " et " des possibles " évoluent en fonction des transformations du contexte économique, juridique, politique et social.

\section{L'immigration féminine latino-américaine en Belgique}

Contrairement à l'immigration " politique " des années 1960 et 1970, I'immigration dite "économique " latino-américaine vers l'Europe connaît une temporalité différente. Alors que l'immigration des pays hispanophones est devenue numériquement importante dès les années 1990, l'immigration brésilienne n'a pris de l'ampleur que vers le début des années 2000 (OIM, 2009). Pour les deux groupes en revanche, il est possible d'identifier une intensification sensible des flux vers la Belgique (et d'autres pays européens) pendant les années 2000.

Plusieurs facteurs interviennent dans cet accroissement de la migration latino-américaine, en particulier féminine, vers la Belgique. Un premier élément concerne la politique de visa menée par les pays de l'espace Schengen. Comme le souligne Jean-Yves Carlier (2008), au moment de la signature de l'Accord Schengen (1985), I'Espagne a réussi à négocier la non-inclusion des pays d'Amérique latine dans la liste des pays demandant un visa à l'entrée. Le changement de législation en 2002 et 2003, avec la mise en place d'un visa pour l'Équateur et la Colombie, a ralenti drastiquement ces flux en direction de l'Europe. A contrario, la non-exigence de visa pour les Boliviens explique pourquoi leur nombre a augmenté, du moins jusqu'en 2007, date d'introduction du visa obligatoire. Les Brésiliens, pour leur part, sont encore exemptés de l'obligation du visa d'entrée. Cette facilité relative constitue de fait une variable importante pour expliquer le choix de certains pays européens, dont la Belgique, comme pays de destination. Un grand nombre des migrants latino-américains sont donc entrés légalement en Europe, munis d'un visa touristique à l'entrée sur le territoire. Dans la majorité des cas, ces migrants ne sont pas retournés dans leur pays d'origine à l'issue des trois mois de séjour légal et ont donc prolongé leur séjour de manière irrégulière devenant de la sorte des overstayers.

La population brésilienne résidant légalement en Belgique est passée de 1718 personnes en 2000 à 6438 personnes en 2011 (soit 0,3\% de la population étrangère en 2011). On observe que les femmes sont plus nombreuses que les hommes malgré une relative diminution au cours des dernières années (passant de $67,05 \%$ en 2000 à $61,6 \%$ en 2010). Selon une étude réalisée par l'Organisation Internationale des Migrations (2009), les estimations du nombre de Brésiliens en situation irrégulière varient entre 10000 et 50000 individus. En ce qui concerne la population équatorienne (groupe le plus important parmi les femmes origi- 
naires des pays hispanophones), une étude datant de $2003^{3}$ évalue leur nombre entre 15000 et 20000 ; cette population réside majoritairement à Bruxelles et à Anvers, villes où le marché du travail informel est le plus développé. Cependant, les derniers chiffres officiels émanant du Registre national (2011) indiquent que la communauté équatorienne résidant légalement en Belgique est constituée de 5137 personnes (dont 1779 ont acquis la nationalité belge).

Depuis les années 1990, Le Brésil, outre l'expansion et la consolidation de la classe moyenne et l'achèvement d'un long processus d'urbanisation, d'industrialisation et d'ouverture du pays aux marchés internationaux, connaît des changements importants touchant la structure même de la famille alors que les femmes semblent y avoir un rôle relativement plus prépondérant ${ }^{4}$. Cette évolution est plus sensible dans les régions économiquement plus actives du centre-sud et dans la classe moyenne à laquelle appartient la grande majorité des migrants brésiliens de Belgique (OIM, 2009 : 43-44) - et particulièrement les femmes qui ont participé à notre étude. Celles que nous avons rencontrées se présentent comme les " entrepreneurs " de leurs trajets migratoires et l'immigration est considérée comme une "opportunité " ou un "pari d'avenir " - un choix fait parfois contre l'avis de leurs familles et entourage.

Une partie importante des femmes hispanophones participant à notre enquête sont originaires des villes moyennes de l'Équateur et dans une moindre mesure de Bolivie et de Colombie, considérablement touchées par la pauvreté et le chômage résultant des différentes crises qui ont frappé l'économie de ces pays. II n'est donc pas étonnant qu'elles soient aussi les plus touchées par la migration, en particulier des femmes, vers l'Europe et les États-Unis (Padilha, 2006). Il s'agit bien souvent de femmes vivant dans des structures sociales où la famille occupe une place importante - et où la femme est soumise à un plus grand contrôle social. L'immigration est pour elles bien souvent un moyen d'échapper à leur situation, mais c'est aussi une décision qui est prise au sein même du groupe familial. Dans le cas des migrations latino-américaines hispanophones, on assiste davantage à une " homogénéisation genrée des profils migratoires ". Les difficultés que les hommes rencontrent en matière de travail pousseraient alors les femmes à aider d'autres femmes à migrer plutôt que des hommes.

\section{La place des femmes latino-américaines dans le " marché du care " belge}

Comme Helma Lutz l'a observée, la féminisation des flux migratoires vers l'Europe est le résultat de l'agencement de trois " régimes " : un "régime

\footnotetext{
3 Unité de psychologie sociale et des Organisations, Louvain-La-Neuve, 2003, in Walter Andino et Sabine Craenen (2009) L'Équateur ici et là-bas, Rapport du Projet "Droits au travail des migrants hispanophones vivant en Belgique ", Bruxelles, OR.C.A., p 3.

4 Malgré ce processus, il est important de noter que les inégalités dont les femmes sont victimes restent bien présentes au Brésil, ainsi que l'acceptation (et reproduction) du partage inégal des tâches qui relèvent du " care " (tâches domestiques, éducation des enfants, etc.), même dans les classes moyennes. Selon une étude de I'IBGE (2006), 92 \% des femmes brésiliennes professionnellement actives seraient ainsi également responsables du travail domestique - contre seulement $0,8 \%$ des hommes - et y consacreraient vingt-cinq heures par semaine en moyenne.
} 
genré ", caractérisé par la naturalisation des pratiques culturelles qui assignent aux femmes les tâches domestiques; un "régime du care " qui concerne les systèmes de " welfare " et les politiques publiques qui distribuent la responsabilité des soins et de l'aide entre l'État, la famille et le marché ; et enfin un "régime migratoire " relatif aux politiques et institutions qui régulent l'entrée, la circulation et l'insertion des migrants dans les pays d'accueil (Lutz, 2008 : 16-17). De manière évidente, les contraintes et les opportunités qui dépendent de l'imbrication de ces trois régimes contribuent à façonner les carrières des femmes latino-américaines en Belgique. Leur agencement spécifique dans ce pays, et en particulier le régime migratoire, explique la place importante qu'occupe le secteur de la domesticité informelle.

Premièrement, il existe très peu de possibilités légales de migrer pour travailler dans le secteur de la domesticité en tant que ressortissant d'un pays tiers à l'Union européenne. Outre le secteur diplomatique ou le travail en tant que fille au pair, la seule possibilité prévue par la loi consiste en l'obtention d'un permis de travail $B$ (valable pour une période d'un an/renouvelable uniquement auprès du même employeur). Ce permis a pour objectif premier de permettre à des étrangers d'occuper des positions sur le marché de l'emploi belge à condition qu'il n'y ait personne qui puisse satisfaire à la demande (selon le principe de préférence communautaire). Un examen du marché du travail par les administrations régionales doit être réalisé avant d'octroyer ou non une autorisation $d^{\prime}$ occupation à un travailleur étranger ${ }^{5}$. La non-reconnaissance de " carence en main-d'œuvre " du secteur de la domesticité par les pouvoirs publics ${ }^{6}$ le ferme aux travailleurs étrangers et finit par renforcer l'informalité des relations de travail (Godin, 2013). En effet, les chiffres relatifs au nombre de permis B délivrés dans ce secteur indiquent que I'utilisation de cette filière par les employeurs est marginale. De plus, ces permis sont uniquement délivrés par les administrations régionales dans le cas d'un travail domestique effectué uniquement en tant qu'interne ("live-in ") et non en tant qu'externe ("live-out "), dans la mesure où l'on considère qu'il n'y a personne sur le marché de l'emploi belge pouvant occuper la fonction (OR.CA, $2010: 26)$.

Deuxièmement, en juillet 2001, le gouvernement belge a tenté de formaliser le secteur domestique par la mise en place du système de titre-service. Soutenu par l'État et renforcé en 2004, il a été prévu au départ pour promouvoir la réintégration des chômeurs de longue durée. Ce système est basé sur une relation triangulaire entre le travailleur et l'employeur (une société intermédiaire

\footnotetext{
5 De plus, pour obtenir un permis B, le travailleur doit être ressortissant d'un pays avec lequel la Belgique est liée par une convention ou un accord international en matière d'occupation des travailleurs. Il existe aussi un certain nombre d'exceptions spécifiées dans la loi pour lesquelles l'octroi d'un permis B est facilité, ne requérant aucune condition particulière, comme par exemple le personnel hautement qualifié ou les postes de direction (cf. article 9 et chapitre 6 de l'arrêté royal du 09/06/1999 portant exécution de la loi du 30 avril relative à l'occupation des travailleurs étrangers).
}

$6 \mathrm{La}$ reconnaissance officielle de " carence de main-d'œuvre " dans un secteur spécifique lève I'obligation, pour I'administration, de vérifier au préalable l'inexistence de travailleurs disponibles localement pour I'octroi des permis de type B. 
dite entreprise de "titre-service $\left.{ }^{7}\right)$, I'employeur et l'utilisateur du système de chèques, et le travailleur et I'utilisateur. II repose sur une déduction fiscale et a été soutenu par les syndicats. Alors qu'il a connu un succès grandissant au cours des dernières années (il est passé de 61759 travailleurs en 2006 à 149827 travailleurs en 2011) (IDEA Consult, 2012 : 15), on observe cependant que son taux de pénétration est moins important dans la Région de Bruxelles-Capitale et ce, comparativement aux deux autres Régions du pays ${ }^{8}$. En outre, une enquête récente, menée par la Gesellschaft für Angewandte Wirtschaftsforschung $\mathrm{mbH}$ en 2010, a montré que les employeurs continuent d'embaucher des travailleurs de manière illégale. Différents facteurs peuvent expliquer cette situation malgré les avantages (apparents) offerts par le système des titres-services. Tout d'abord, beaucoup de travailleurs pour attirer les clients vendent leurs services à des taux horaires inférieurs à ceux pratiqués sur le marché légal de la domesticité ${ }^{9}$, ensuite ce système, qui recense les activités légales (nettoyage, lavage des vitres, lessive, repassage et occasionnellement cuisine et petite couture) ne prévoit pas la garde des enfants, par exemple. Or, la majorité des femmes latino-américaines que nous avons rencontrées combine régulièrement les tâches domestiques avec celles liées à la garde des enfants. Enfin, le nombre de chèques octroyés par " unité familiale " est limité ( 1000 chèques par ménage par an en 2012), ce qui contribue également à augmenter le travail illégal. En réalité, comme certaines femmes enquêtées le laissent entendre, nombreuses sont celles qui travaillent pour un employeur unique en tant qu'interne ou en tant qu'externe et pour lequel elles effectuent des tâches liées au care (nettoyage, garde des enfants, jardinage, soins, etc.). Ces cas de figure font cependant l'objet de peu d'attention de la part des pouvoirs publics alors que I'on retrouve nombre de femmes sans-papiers dans ce secteur qui viennent le plus souvent d'arriver en Belgique.

Troisièmement, le secteur de la domesticité en Belgique s'inscrit dans un « jeu de chaises musicales " (game of ethnic musical chairs) bien connu (Waldinger, 1994) où les plus anciennes immigrantes sont remplacées par les plus récentes. Après la chute du mur de Berlin et la suppression du visa d'entrée en 1991, le secteur de la domesticité informelle à Bruxelles a été principalement occupé par des nouvelles migrantes polonaises (Kuźma, 2012). Depuis l'entrée de la Pologne au sein de l'Union européenne et surtout, depuis la levée des restrictions en matière d'accès au marché du travail belge (1er mai 2009), les migrantes polonaises ont été remplacées, partiellement, par des femmes en provenance des pays récemment adhérents à I'Union européenne (Bulgarie et Roumanie) et des

7 Différents types de sociétés existent : certaines font partie de l'économie sociale, d'autres sont des sociétés commerciales, et enfin, d'autres encore appartiennent au secteur public. Grâce à cette gamme d'acteurs, le secteur a été défini comme un quasimarché avec un niveau élevé de concurrence entre les entreprises (commerciales ou autres) (Henry et al., $2008: 5$ ).

8 En 2011, Bruxelles avait un taux de pénétration de 9,2 \% (76 889 utilisateurs pour une population de 838601 ) alors que la Région flamande avait un taux de 10,3\% (504 259 utilisateurs pour une population de 4915 523) et la Région wallonne de 9,5 \% (253 811 utilisateurs pour une population de 2678 811) (Population $\geq 20$ ans) (IDEA Consult $2012: 17)$.

9 Grâce aux subsides du gouvernement fédéral, le Titre-Service, d'une valeur de 22,04 € par heure de prestation, a une valeur d'achat de 8,50€ pour le particulier. Depuis le 1 er janvier 2013, après déduction fiscale, le coût réel pour l'utilisateur par titre-service est de 5,95€ (http://www.titres-services-onem.be/). 
pays d'Amérique latine et d'Asie (essentiellement des Philippines). Ainsi, la structuration du marché du care en Belgique conduit progressivement à une spécialisation ethnique et genrée avec d'un côté, des femmes issues des nouvelles migrations en provenance du Sud (essentiellement d'Amérique latine et d'Asie), qui occupent majoritairement le secteur informel et de l'autre côté, des femmes provenant des pays de l'Union européenne, ainsi que celles issues des plus veilles migrations (comme le Maroc ou la République Démocratique du Congo) plus présentes dans le circuit formel du marché de la domesticité.

\section{Analyse des carrières migratoires}

\section{Remarques méthodologiques}

Le travail de terrain a été effectué à Bruxelles entre juin 2009 et mars 2010. Vingt-deux entretiens semi-directifs ont été conduits avec des migrantes latinoaméricaines (majoritairement en séjour irrégulier au moment de l'enquête) qui travaillaient dans le secteur de la domesticité. Outre les entretiens individuels, deux "focus groups " ont été réalisés, l'un portait sur les migrations des pays hispanophones (majoritairement originaires de l'Équateur) et l'autre sur l'immigration féminine brésilienne.

Les femmes ayant participé à la recherche sont représentatives de l'immigration brésilienne vers I'Europe (OIM, 2009 : 43-44) : toutes étaient issues de la classe moyenne de villes des États du centre-sud brésilien (principalement, Minas Gerais, Goiás et Distrito Federal). Le niveau de scolarité correspondait à la fin du secondaire. La grande majorité était employée au moment d'immigrer. Les femmes hispanophones enquêtées étaient majoritairement originaires des villes moyennes de l'Équateur (Santo Domingo, Otavalo, Ambato, Esmeraldas, Loja, Cuenca, Cañar, Machala).

II est important de souligner que notre recherche s'est déroulée lors de la deuxième campagne de régularisation lancée par le gouvernement belge le 18 juillet 2009. Elle a permis à nombre de nos interviewées d'introduire une demande de régularisation qui n'a pas été sans effets sur nos résultats concernant l'évolution des projets et carrières des femmes latino-américaines concernées.

Nous avons aussi participé durant cette campagne qui s'est déroulée du 15 septembre 2009 au 15 décembre 2009 aux activités de l'association d'aide aux migrants "Abraço " et assistées aux permanences de la Confédération des Syndicats Chrétiens CSC. Nous avons pu ainsi entrer en contact avec certaines des femmes enquêtées.

L'analyse thématique des discours a été menée avec pour cadre théorique les problématiques liées au concept de " carrière migratoire " qui permet de rendre compte des évolutions, changements et bifurcations dans la vie des femmes ainsi que leurs projets et souhaits depuis le pays d'origine jusqu'au moment de l'entretien. En effet, les femmes ne sont pas dépourvues d'agency les conduisant à répondre aveuglement aux demandes d'un marché global du care ou à un système genré de partage des tâches. De même, leurs carrières ne sont pas uniquement façonnées par des politiques migratoires restrictives. Le concept de 
" carrière migratoire " permet ainsi de comprendre l'évolution des projets migratoires des femmes comme faisant partie d'un processus dynamique dans lequel des facteurs objectifs (contexte politico-institutionnel, économique) et subjectifs (attentes de départ, réalités vécues, expérience migratoire) s'entremêlent. Sa portée heuristique se fonde sur la conjonction de trois niveaux d'analyse : les caractéristiques individuelles des acteurs (niveau micro), la mobilisation des ressources et du réseau social (niveau méso) et la structure des opportunités et des contraintes des migrations internationales (niveau macro) (Martiniello et Rea, 2011).

\section{Les différentes " modalités " d'entrée dans la domesticité}

À partir de nos données empiriques, nous avons pu identifier trois portes d'entrée dans le marché du travail domestique (irrégulier) belge. Trois " modalités " s'imposent : une première, dite de " cooptation "; une deuxième dite " entrepreneuriale " et la troisième, que nous appelons " intermédiaire ". Dans l'agencement de chacune de ces modalités, il est possible d'observer comment les femmes mobilisent leurs ressources personnelles ${ }^{10}$ pour trouver une place sur le marché de la domesticité. Si ces modalités se rapportent, dans un premier temps, aux différentes façons d'entrer en domesticité, dans un deuxième temps, elles permettent de mieux comprendre les changements qui surviennent en cours de parcours et qui façonnent les carrières des femmes dans leur globalité. Loin d'être linéaires, ces carrières connaissent des bifurcations qui sont le résultat de changements liés aux opportunités socio-politiques, à la capacité à mobiliser des ressources consécutives à l'acquisition de nouveaux capitaux et aux trajectoires individuelles. Les évolutions des carrières, parfois accompagnées d'un changement de statut, transforment le projet, qui évolue selon une " confrontation entre les attentes de départ et les réalités vécues au travers de l'expérience migratoire " (Martiniello et Rea, $2011: 2$ ).

La modalité de " cooptation " repose sur l'existence d'un réseau social fort composé de femmes qui mettent en relation des migrantes anciennement ou récemment arrivées et de futurs employeurs (des femmes généralement). Ce sont bien souvent "les pionnières " qui servent de "pont " entre les candidates au départ et leurs futures employeuses en Belgique. Elles organisent le voyage et l'accueil après l'établissement d'un contrat reposant essentiellement sur la confiance. Parce qu'il y a fréquemment une contrepartie à l'aide fournie, une mise au travail rapide de la nouvelle venue se constate régulièrement. Ce processus de cooptation a pour conséquence de renforcer une sélectivité genrée de la migration latino-américaine vers la Belgique.

Il est intéressant de souligner que cette modalité d'entrée a été davantage constatée parmi les femmes équatoriennes, dont la grande majorité est arrivée au début des années 2000, avant le changement de la politique de visa en 2003. Dans le groupe des femmes brésiliennes, elle n'a été rencontrée que chez deux femmes dont I'arrivée en Belgique date du début des années 2000. L'extrait suivant illustre les mécanismes de " cooptation ". II s'agit d'une femme brésilienne de Goiás qui est arrivée en Belgique en 1999, à 23 ans :

10 Par ressources, on entend une configuration particulière de différents types de capitaux (capital social, économique et culturel) au sens de Bourdieu (1997). 
"C'est ma copine qui m'a trouvé mon premier travail, j'étais interne chez une "madame". [...] Je pense que c'était une de ses "madames" qui lui a demandé si elle ne connaissait pas quelqu'un... alors, elle lui a parlé de moi, parce qu'elle savait que je voulais venir. [...] Alors, j'ai acheté mon billet, j'ai pris l'avion et tout... bref, je suis venue et tout de suite après j'ai commencé à travailler. J'ai bien aimé ce travail, la famille m'aimait bien, ce n'était pas difficile. Mais j'ai du arrêter parce que la famille est partie du pays... j'étais un peu triste, ça faisait presque deux ans, tu vois. Alors, à partir de ce moment, j'ai commencé à chercher "des heures" chez l'une, chez l'autre... et j'ai fini par trouver mes "madames"... j'en ai six, les mêmes, ça fait déjà presque... huit ans, je

pense ".

Bien que l'extrait ci-dessus fasse état de l'expérience d'une Brésilienne, il semble cependant que des formes de compétition existent dans les réseaux brésiliens, ce qui permettrait de comprendre pour partie la moindre importance des stratégies de cooptation à la différence de ce que l'on observe chez les femmes équatoriennes. II ne s'agit bien évidemment pas d'avancer l'idée que les femmes brésiliennes sont culturellement moins " solidaires " que les latino-américaines. II semble néanmoins que les relations sociales à l'intérieur de la communauté brésilienne soient marquées davantage par la concurrence, conduisant certains auteurs à parler de "communauté de rivaux " où la méfiance et la compétition finissent par nuire à la solidarité et à l'entraide entre migrants (Rosenfeld, Marcelle et Rea, 2010). Outre ce point de vue, il semble aussi que le moment d'entrée dans le secteur de la domesticité informelle fournisse un élément de compréhension des différences entre femmes brésiliennes et équatoriennes : un certain nombre de femmes équatoriennes interviewées sont arrivées en Belgique entre 2000 et 2003, à un moment où le secteur était relativement moins " compétitif " qu'après l'introduction des titres-services en 2004.

La seconde modalité d'entrée dans le secteur de la domesticité est liée à un comportement de type entrepreneurial. Elle repose davantage sur I' "agency " de la femme migrante, sur ses ressources individuelles et sur sa capacité à " entreprendre ": annonces dans les journaux ou des magasins, distribution de cartes dans les zones résidentielles, etc. Cette modalité d'entrée, que l'on rencontre beaucoup plus chez les femmes brésiliennes possédant un niveau d'éducation élevé et qui travaillaient dans le secteur des services au pays d'origine, correspond davantage à un travail d'indépendant où la travailleuse va tenter de se faire sa propre clientèle. C'est le cas de cette jeune brésilienne, infirmière de formation :

" Je cherchais, je cherchais et comme je ne trouvais rien, j'ai demandé à ma copine : "où est-ce qu'on a le plus besoin de femme de ménage ici ?"; elle m'a dit: "ben, si tu vas à Waterloo, il y a souvent des gens qui demandent". Alors, j'ai fait ça. J'ai écrit moi-même les annonces... avec mon français de l'époque, tu imagines ! [Elle rit] et puis je suis allée là-bas les distribuer, dans les commerces, les boîtes aux lettres... Et c'est comme ça que j'ai trouvé mon premier travail, c'était pour être "interne" dans une famille... parce que je veux être indépendante ! [...] Parce que je vois beaucoup de gens ici [des Brésiliens] qui ne font pas d'efforts pour faire les choses par soi-même. [...] Ils sont toujours en train de te demander de faire ceci, cela. [...] Ils n'ont pas d'ambition ou l'envie de devenir plus autonome [...] je ne comprends pas !». 
Cette jeune femme, qui a choisi de se débrouiller par elle-même, porte un regard critique sur les Brésiliens qui se tourneraient vers leur "communauté " dès qu'ils sont confrontés à des difficultés ou ont besoin de l'aide d'un traducteur pour un rendez-vous médical ou pour rencontrer un nouvel employeur. Dans l'entretien elle nous dira aussi que le recours à l'aide intra-communautaire serait presque systématique chez certaines Brésiliennes, ce qui renforcerait leur lien de " dépendance " vis-à-vis d'une communauté caractérisée pourtant comme " peu solidaire ".

Enfin, la troisième modalité repose sur des "structures intermédiaires ", combinant à la fois le recours à un réseau social essentiellement composé de compatriotes et une stratégie d'action individuelle importante. Le réseau de nos enquêtées s'est constitué après la migration notamment à travers l'implication des migrantes dans des églises, la fréquentation du quartier, la participation à des fêtes organisées par des associations. Comme nous le dit l'une d'entre elles, "les frères et sœurs " de l'église deviennent leur point de référence dans le pays d'accueil, ainsi qu'un capital social mobilisable pour trouver un travail, un logement, etc. :

" [L'église] c'est très bien parce qu'on ne se sent pas seule. [L'église] c'est le lieu où on va pour chercher Dieu, n'est-ce pas? Mais c'est aussi le lieu où je rencontre un support. Parce que les gens sont là pour vous aider aussi. Quand j'ai eu besoin, j'ai toujours pu compter sur eux. [...] Mes premières "madames", ce sont des gens de l'église qui m'ont aidée à les trouver. [...] J'étais ici, mais je ne trouvais rien, c'était difficile [...] j'avais des petites heures par-ci, par-là... mais petit à petit, j'ai réussi [...] les gens [de l'église] savaient que je cherchais, alors ils m'indiquaient des gens".

\section{Les changements liés à la trajectoire de travail dans le secteur de la domesticité}

L'approche par " modalité d'entrée " permet d'avoir une vision plus nuancée de la domesticité informelle, niche économique occupée par les femmes sanspapiers latino-américaines. Dans la littérature scientifique, on distingue le plus souvent la sphère dite du "live-in " où les risques d'exploitation sont les plus importants pour les femmes, et celle du "live-out"(Anderson, 2000). Loin de nier les situations d'exploitation et de discrimination, cette approche binaire ne rend cependant pas compte de l'aspect dynamique du secteur (souvent marqué par le passage constant d'une sphère à l'autre), ni des profils socio-économiques diversifiés des femmes concernées. En spécifiant les " trois modalités d'entrée " des femmes dans les secteurs de la domesticité informelle, notre démarche vise à tenir ensemble, dans l'analyse de leurs trajectoires migratoires, non seulement les caractéristiques des femmes avant qu'elles ne migrent, mais aussi les processus d'acquisition de nouveaux types de capitaux en cours de parcours.

Même si les " modalités " décrites peuvent mener aussi bien à des situations de live-in que de live-out, la modalité d'entrée par "cooptation " destine plus fréquemment à des situations de live-in alors que la modalité d'entrée selon le mode " entrepreneurial " mène davantage à des situations de live-out. Enfin, la troisième modalité, celle des "structures intermédiaires " est plus rarement choisie en début de parcours, puisqu'elle requiert l'existence de contacts dans le pays d'accueil, mais elle permet le plus souvent de passer de situations de domesticité interne à des situations de domesticité externe. 
Les " modalités d'entrée " telles que nous les avons spécifiées nous permettent également de mieux saisir les liens entre des changements dans la trajectoire de travail et la façon dont les femmes redéfinissent leurs projets migratoires ainsi que leurs "stratégies de séjour "(Engbersen, 1999). L'acquisition d'un capital social plus diversifié où " liens forts " et " liens faibles " se renforcent (Granovetter, 1983) rend possible dans une certaine mesure, une forme de "mobilité sociale marginale " dans le secteur d'emploi. Au fil des années, les femmes accumulent progressivement des ressources leur permettant de jongler avec plusieurs types de capital social, construit tant à l'intérieur (" liens forts ") qu'à l'extérieur de la communauté (" liens faibles "), ce qui leur permet d'améliorer leur position sociale sur le marché de la domesticité mais plus largement encore au sein de la société.

L'affaire "Cleanse-Brasileuro ", dans laquelle un certain nombre de nos interviewées se sont trouvées impliquées, permet d'illustrer notre propos. Au début du mois d'avril 2009 des perquisitions ont eu lieu dans les bureaux de l'entreprise de titres-services Cleanse-Brasileuro et le pasteur évangélique, à sa tête, s'est vu inculpé pour escroquerie, détournement de fonds, blanchiment d'argent et emploi de travailleurs en situation irrégulière. Les travailleuses originaires du Brésil et de l'Équateur, bien que sans-papiers, étaient employées avec le système titres-services, dans l'espoir d'obtenir à terme une régularisation par le travail. Alors qu'elles étaient toutes en situation irrégulière, elles avaient cependant acquis un statut de "quasi légalité ", cotisant auprès de la sécurité sociale belge. Si les femmes rencontrées, tant Brésiliennes qu'Équatoriennes, avaient choisi de s'impliquer dans cette entreprise, c'était d'abord parce qu'elles faisaient confiance aux "frères " et " sœurs " de la même église. Cette affaire cependant n'a pas été sans effets sur leurs aspirations, du moins pour certaines d'entre elles, qui ont mesuré l'avantage d'avoir un travail déclaré et ont tenté de régulariser leur séjour mettant à profit la médiatisation de l'affaire et l'arrestation du prêtre. En attendant la régularisation de leur séjour, la plupart des migrantes ont pu retrouver la situation qu'elles avaient avant d'entrer dans l'entreprise de titres-services, retournant pour la plupart travailler dans le secteur de la domesticité informelle chez leurs employeurs respectifs.

\section{La campagne de régularisation dans l'évolution des " carrières migratoires "}

En Belgique, une deuxième campagne de régularisation ${ }^{11}$ a été lancée le 18 juillet 2009 sur la base à la fois de critères de régularisation permanents (longue procédure, situations humanitaires urgentes, famille avec enfant scolarisé) et temporaires ${ }^{12}$. Parmi les critères temporaires figuraient le critère de l' « ancrage

\footnotetext{
11 La première grande campagne de régularisation dite one-shot a vu le jour grâce à la loi du 22 décembre 1999 relative à la régularisation. À cette époque, 32662 personnes et/ou familles en séjour illégal ou précaire ont eu la possibilité de s'établir de manière permanente et complète dans notre pays, moyennant le dépôt d'une demande de régularisation auprès de l'administration communale de leur domicile au cours du mois de janvier 2000 (Centre pour l'Égalité des Chances et Lutte contre le Racisme (2008) Before \& After, [en ligne]. URL : http://www.ulb.ac.be/socio/germe/documentsenligne/BAfr.pdf).

12 L'instruction relative à I'application de I'ancien article 9, alinéa 3 et de l'actuel article 9 bis de la loi du 15 décembre 1980 sur les étrangers.
} 
local durable " (2.8.A) et celui de la " régularisation par le travail " (2.8.B ${ }^{13}$. Les demandes de régularisation dans le cadre des critères temporaires devaient être introduites entre le 15 septembre 2009 et le 15 décembre 2009. Dans le cadre de la régularisation par le travail, le candidat devait prouver qu'il avait continuellement résidé en Belgique depuis le 31 mars 2007 et fournir une copie du contrat de travail signé avec l'employeur (soit à durée déterminée, soit à durée indéterminée).

Si au premier abord, ce critère semblait ouvrir des perspectives réelles pour les travailleurs en situation irrégulière résidant depuis peu en Belgique, la complexité administrative de la procédure et le manque de confiance dans les institutions, tant du côté des personnes candidates à la régularisation, que du côté des employeurs potentiels, $\mathrm{n}^{\prime}$ a pas permis une régularisation massive par le travail. C'est le critère de l'u ancrage local durable " qui a davantage été privilégié par les migrants en situation irrégulière comme ce fut le cas de la majorité des femmes que nous avons rencontrées. II s'agissait d'apporter la preuve d'une présence ininterrompue d'au moins cinq ans avec soit un titre de séjour, soit des tentatives crédibles de l'obtenir avant le 18 mars 2008. Ce critère offrait également davantage de garanties conférant ainsi au demandeur un titre de séjour permanent (renouvelable) donnant accès au marché du travail belge ${ }^{14}$.

Nos entretiens ont indiqué que les femmes hispanophones ont fait de la régularisation un de leurs buts principaux qu'elles ont essayé d'atteindre de façon plus soutenue que les femmes brésiliennes qui ne sont pas soumises à la même politique de visa que les autres femmes latino-américaines. Pour celles qui ont obtenu une régularisation et les propos recueillis en témoignent, leur situation socio-professionnelle s'est largement améliorée. Si les " carrières " se modifient en fonction de parcours juridico-institutionnel et socio-économique, nos entretiens mettent aussi en évidence, d'un point de vue subjectif, comment des projets de départ se transforment en fonction des expériences vécues et de la façon dont les individus perçoivent leur propre identité. Pour les femmes hispanophones, la " stratégie de séjour " (Engbersen, 1999) par la régularisation, qui au départ du parcours migratoire n'était pas un objectif, devient d'une certaine façon, le moyen de briser la " chaîne du care " dont elles se sentent souvent " prisonnières".

Le concept de " global care chain ", tel que le définissent Barbara Ehrenreich et Arlie Russell Hochschild (2002), fait référence à la chaîne qui se crée lorsqu'une femme d'un pays en développement part vers un pays développé pour assumer la responsabilité des "soins " qu'une femme de ce pays ne peut/veut pas assumer, tout en assignant à une autre femme, restée dans son pays d'origine, la place qu'elle-même laisse vide à cause de son départ. Les auteurs dénoncent ainsi la perte de capital humain (féminin) dans les pays en développement en raison de cette dynamique transnationale. Néanmoins, certaines configurations

\footnotetext{
13 La majorité des demandes a été traitée dans le courant de 2010 et 2011, avec respectivement 24999 et 9509 personnes régularisées. Cependant, il n'est pas possible de distinguer les demandes liées à l'instruction de celles liées aux demandes " ordinaires" à partir des données publiées par I'Office des Étrangers (Centre pour l'Égalite des Chances et la Lutte contre le Racisme, 2012 : 120).

$14 \mathrm{Via}$ la régularisation par le travail, les candidats se voyaient délivrer une autorisation de séjour d'un an - renouvelable - lié à un permis de travail B.
} 
de cette " global care chain " peuvent aussi se mettre en place dans le pays d'immigration. Pour les femmes en situation irrégulière, avec des enfants en bas âge, avoir accès aux crèches publiques est quasiment impossible, ce qui les conduit à adopter des "stratégies d'évitement " les amenant à trouver des solutions à l'intérieur de leur cercle familial. Ainsi, des grands-mères peuvent s'improviser "gardiennes " afin de permettre à la plus jeune génération de travailler ou encore d'en retirer une aide financière. La venue régulière ou irrégulière des grands-mères en Belgique, permet ainsi à certaines femmes de rester disponibles aux horaires qui conviennent à leurs employeuses.

La situation est différente lorsque les enfants sont restés au pays d'origine. Bien que l'ensemble des femmes latino-américaines soit soumis aux mêmes difficultés pour obtenir un permis de travail dans le secteur de la domesticité ou un titre de séjour, les femmes brésiliennes, exemptées de visa d'entrée, jouissent d'une plus grande (bien que relative) mobilité, qu'elles mettent à profit pour accueillir leurs enfants - mais aussi leurs conjoints et autres membres de leurs familles. Cette possibilité leur donne le sentiment d'une " connexion permanente " avec les enfants restés au pays d'origine.

En revanche, les femmes hispanophones sont plus souvent contraintes de rester en Belgique (le retour au pays d'origine pouvant mettre en péril leur séjour dans le pays d'accueil) et donc de vivre éloignées de leurs enfants, sous la garde d'une autre femme de leur famille au pays. La majorité de celles que nous avons rencontrées n'a pas anticipé le changement de législation en matière de visa survenu en 2003 qui a eu pour conséquence de séparer des familles pour bien plus longtemps que prévu, comme l'illustre l'extrait suivant:

" $R$ : quand on a décidé de partir, on a dit pour trois ans, ça suffira et on rentrera mais... après c'était fermé. [...] I : quel âge avait votre fille quand vous êtes partie ? $R$ : trois ans et demi, maintenant elle a treize ans, elle habitait avec ma maman ; ma maman l'année dernière elle est morte donc ma fille elle reste avec une sœur qui est encore là et avec mon papa. Ça lui permet d'aller à l'école et d'avoir des meilleures choses que moi. I: et pourquoi vous n'êtes pas venue directement avec votre fille? F : c'était juste pour trois

ans $"$.

Les différentes " stratégies de séjour " entre les deux groupes de femmes sont visibles quand on observe le nombre important de procédures de régularisation entreprises par les Latino-américaines hispanophones avant la campagne de régularisation de 2009. Pour les Brésiliennes, cette campagne a d'abord été une façon de sortir de l'ombre, ce qui ne veut pas dire qu'elles ne souhaitaient pas être régularisées mais dans les entretiens menées avec elles, la régularisation n'est pas leur principale priorité, certaines restant très attachées à leur projet de départ, envisagé avant tout comme un investissement (voir infra). Cependant, pour les deux groupes de femmes, le fait d'avoir des enfants en Belgique renforce le souhait de régulariser leur situation pour qu'ils puissent accéder à une scolarité normale. Comme lors d'une étude antérieure (Godin et Rea, 2010), on soulignera ici le rôle joué par certains employeurs qui aident les migrantes dans leurs démarches de régularisation.

En somme, l'engagement dans cette "stratégie de séjour " est étroitement lié à la trajectoire dans la sphère du travail, au type de réseau social que les 
femmes ont pu développer, aux événements biographiques survenus dans leur vie (la séparation forcée avec ses enfants restés au pays d'origine ou la présence des enfants en Belgique) et enfin, aux structures d'opportunités politiques (comme la fermeture des frontières et la campagne de régularisation de 2009). II y a donc une imbrication constante des trois niveaux d'analyse (macro, méso et micro) qui permet de saisir les carrières des femmes.

\section{" Projets " et « carrières migratoires "}

Les femmes latino-américaines que nous avons rencontrées évoluent souvent d'un projet migratoire initial " d'investissement " vers un projet "d'installation " en Belgique (Van Meeteren, Engbersen et Van San, 2009). On soulignera cependant que, contrairement à ce que laissent penser ces auteurs, la régularisation n'est pas en soi une " aspiration ", ni un projet, mais plutôt un " moyen " que les migrantes utilisent pour contourner les contraintes liées à leur statut. Ils proposent une triple classification des migrants, basée sur leurs " aspirations " et la manière dont ils mobilisent leurs capitaux (culturel, social et économique) afin de les réaliser. Sur la base d'une enquête menée en Flandre et à Bruxelles, les auteurs identifient "trois types d'aspiration " qui correspondent à " trois types de migrants ". Le premier, les migrants investisseurs (Investment migrants), " aspirent " principalement au retour une fois réalisé leur ambition d'épargne. Le second, les migrants en voie d'être régularisés (Legalizing migrants), " aspirent " à "l'obtention de papiers ", pour régulariser leur séjour dans le pays d'accueil. Enfin, le troisième, les migrants "qui s'installent " (Settlement migrants), " aspirent " à s'établir et résider définitivement dans le pays d'accueil, en dépit de leur statut juridique (régulier/irrégulier).

Nos propres recherches montrent que si nombre de femmes ont comme projet de départ le souhait d'émigrer pour travailler et accumuler un capital économique pour ensuite rentrer au pays, avec le temps nombre d'entre elles ont tendance à vouloir s'investir dans un projet d'installation, qui passe par des tentatives de régularisation. Cependant cette évolution ne se fait pas de la même façon pour toutes, ni pour les mêmes raisons. Comme on l'a vu le facteur déclencheur de ce changement peut être l'arrivée des enfants, pour d'autres, ce sera le mariage, ou encore le sentiment d'être plus "en sécurité " (autant physique que financière) et/ou d'avoir une meilleure "qualité de vie " (travail plus gratifiant, accès aux soins, logement, etc.) dans le pays d'accueil. De plus, le capital social accumulé en Belgique devient plus " adapté " à la société d'accueil que celle d'origine, ce qui finit par réduire les possibilités de réinsertion socioprofessionnelle au pays d'origine. Mais nos entretiens ont montré que rien n'est acquis et en fonction des hasards et des transformations du contexte (maladie d'un proche, chômage, difficulté à trouver un logement, etc.) les femmes peuvent également réorienter leur projet vers un retour au pays d'origine.

La reconfiguration des rapports de genre à l'intérieur des couples en situation post-migratoire peut également conduire à une réévaluation du projet de départ. À cet égard, il est important de mentionner la dépendance des hommes latinoaméricains hispanophones à l'égard du capital social de leur partenaire pour trouver un emploi. Ainsi, alors que les hommes continuent à penser à un retour au pays d'origine et vivent leur situation en Belgique comme temporaire, ce 
sont bien souvent les femmes qui après quelques années n'envisagent plus de rentrer, devenues les breadwinners de la famille. Pour les hommes brésiliens, même si leur insertion dans le marché du travail, notamment dans la construction, se fait plus facilement, une étude de l'OIM (2009) révèle qu'ils font plus souvent appel au programme de retour volontaire que les femmes ${ }^{15}$.

D'autres facteurs, bien que moins souvent énoncés, peuvent inciter les femmes à vouloir régulariser leur séjour, notamment la possibilité de réaliser un changement de statut professionnel. Parmi nos enquêtées, nombre de femmes connaissent un déclassement professionnel et social en travaillant dans la domesticité, notamment celles qui ont un niveau d'éducation élevé. Elles vivent leur trajectoire dans la domesticité comme un passage obligé ; loin d'être un choix, la domesticité s'est plutôt imposée à elles par manque d'opportunités. La " stratégie de séjour " que constitue la régularisation n'est donc pas une aspiration mais un moyen permettant de réaliser d'autres aspirations.

Certaines migrantes mettent en sursis les projets qu'elles avaient au départ, d'autres par contre parviennent à saisir les opportunités et à s'adapter à de nouvelles situations. L'extrait ci-dessous montre comment l'expérience de la mobilisation collective pour le droit des travailleurs migrants sans-papiers au sein de l'entreprise de titres-services Cleanse-Brasileuro a fait naître l'envie de "faire autre chose ", envisageant la régularisation comme une porte de sortie du secteur de la domesticité :

"Écoute avec tous ces problèmes que j'ai eus, je suis devenue presque comme une assistante sociale, j'ai beaucoup aidé les gens, il y a la possibilité de faire une formation comme ça, ou une formation de droit aussi, je sais que... je pense que le droit belge est un peu compliqué, mais que je commence à faire quelque chose, à étudier, je vais commencer à me renseigner auprès des gens, et j'espère. [...] J'ai perdu mon temps, parce que, c'est vrai qu'avant je ne connaissais pas beaucoup de choses et je travaillais toujours, j'étais toujours dans mon travail de la maison et les choses comme ça et c'est maintenant que je commence à me réveiller un peu ".

Comme l'écrivent Marco Martiniello et Andréa Rea (2011), " le hasard conduit parfois à des bifurcations de carrières renforçant le projet initial de l'acteur ou alors le précipitant dans une orientation non souhaitée ". Les carrières migratoires ne sont pas déterminées, elles se construisent aussi en réaction à des événements " imprévisibles ", comme la participation à une action collective et/ ou une campagne de régularisation. En réalité, plusieurs " aspirations " peuvent coexister les unes à côté des autres et elles peuvent devenir plus ou moins prioritaires en fonction des opportunités.

15 Les derniers chiffres sur le retour volontaire depuis la Belgique indiquent qu'entre 2007 et 2011, 5275 migrants originaires d'Amérique latine ont pris part à ce programme. La majorité de ces retours concerne les Brésiliens avec en 2011, 770 Brésiliens sur 881 ressortissants d'Amérique latine (soit $93 \%$ ). On constate également une diminution du nombre d'Équatoriens au fil des années, qui passe de quatre-vingt-onze personnes en 2005 à quinze personnes en 2011. En 2011, I'OIM a assisté 3358 migrants et les Brésiliens constituaient le groupe le plus important (27\% de l'ensemble des demandes) (OIM, 2011). 


\section{Conclusion}

Nous avons proposé dans cet article un nouveau regard sur l'immigration féminine latino-américaine en Belgique. En nous intéressant aux " carrières migratoires ", nous avons mis en évidence la diversité et la complexité des parcours et nous avons montré que ces carrières ne se réduisaient pas à la sphère du travail domestique, bien au contraire. Des liens forts et faibles tissés au cours des parcours, des changements liés aux politiques migratoires et des évènements biographiques spécifiques conduisent à réévaluer le projet de départ et à façonner de manière singulière les carrières migratoires des femmes latino-américaines. Le projet migratoire est soumis aux incertitudes de chaque parcours, aux hasards des rencontres, à l'imprévu des situations vécues, et enfin aux contextes politiques et sociologiques.

Nous avons montré enfin en quoi la catégorisation des projets migratoires ne prenant comme critères que les " aspirations " à un moment donné ne permettait pas une analyse des processus par lesquels ces projets se construisent, et surtout de la manière dont certains aspects structurels les façonnent. Présentés en tant qu'aspiration, l'investissement, la régularisation et/ou l'installation deviennent de simples " ambitions ", construites subjectivement par les acteurs sans rapport explicite avec le contexte social, économique, politique et institutionnel dans lequel elles s'inscrivent, par définition en permanente évolution.

Or, non seulement les projets migratoires se transforment avec le temps, mais également les capitaux dont disposent les migrantes, leurs identités, leurs ambitions et leurs aspirations - des changements souvent étroitement liés. Au lieu de concevoir "l'investissement " et " l'installation " comme des " aspirations-type " invariables et cloisonnées, il est plus judicieux de les appréhender comme des projets qui marquent des "étapes " différentes dans l'évolution des " carrières ". Les femmes migrantes dans leur carrière sont amenées à réajuster en permanence leur conception des possibles.

\section{Références bibliographiques}

Anderson Bridget (2000) Doing the dirty work? The Global Politics of Domestic Labour, London and New York, Zed Books, 224 p.

Bourdieu Pierre (1997) The forms of capital, in Albert Henry Halsey, Hugh Lauder, Phillip Brown and Amy Stuart Wells Eds., Education: Culture, Economy, Society, Oxford, Oxford University Press, pp. 46-58.

Carlier Jean-Yves (2008) Évolution des politiques relatives aux migrations entre l'Amérique latine et l'Europe, in YsabelYépez et Gioconda Herrera Éds., Nouvelles migrations latino-américaines en Europe : Bilans et défis, Louvain-la-Neuve/ Barcelone, Presses universitaires de Louvain (UCL)/Publications I Edicions de la universitat de Barcelona, pp. 289-308.

Centre pour l'Égalité des Chances et la Lutte contre le Racisme (2012) Rapport Annuel Migration 2011, [en ligne] consulté le 16/01/2013. URL : http://www. diversite.be 
Centre pour l'Égalité des Chances et la Lutte contre le Racisme (2008) Before \& After : La situation socio-économique des personnes ayant bénéficié de la procédure de régularisation du 22 décembre 1999, [en ligne] consulté le 16/01/2013. URL : http://www.diversite.be

Ehrenreich Barbara et Hochschild Arlie Russell (2002) The Global Woman. Nannies Maids and Sex Workers in the New Economy, New York, Metropolitan Books, $336 \mathrm{p}$.

Engbersen Godfried (1999) Sans-papiers : Les stratégies de séjour des immigrés clandestins, Actes de la recherche en sciences sociales, 129, pp. 26-38.

Favell Adrian (2009) Eursotars and Eurocities. Free movement and Mobility in an integrating Europe, UK, Blackwell Publishing, 296 p.

Gesellschaft für Angewandte Wirtschaftsforschung mbH (2010) Acceptance and perceived prevalence of the Belgium service voucher system, Research project, 5 February 2010.

Godin Marie (2013) Domestic work in Belgium: crossing boundaries between informality and formality, in Anna Triandafyllidou (Ed.), Irregular Migrant Domestic Workers in Europe: Who Cares?, Ashgate, pp.17-42.

Godin Marie et Rea Andrea (2010) Une analyse genrée de la population de sans-papiers régularisés lors de la campagne de 2000 en Belgique, Migrations Société, 22 (129-130).

Granovetter Mark (1983) The strength of weak ties: A network theory revisited, Sociological Theory, 1, pp. 201-233.

Gutiérrez Elke and Craenen Sabine (2010) Domestic Staff: another look, avec le soutien du Groupe de Recherches Interdisciplinaires sur l'Amérique latine (GRIAL), Université catholique de Louvain (UCL), OR.C.A vzw.

Henry Arnaud, Nassaut Stéphane, Defourny Jacques et Nyssens Marthe (2008) Titres-Services : Régulation quasi-marchande et performances comparées des entreprises prestataires, Revue belge de sécurité sociale, 2, pp. 141-169.

Hochschild Arlie Russell (2000) Global Care Chains and Emotional Surplus Value, in Will Hutton and Anthony Giddens Eds., On The Edge: Living with Global Capitalism, London, Jonathan Cape, pp.130-146.

IDEA Consult (2012) Évaluation du régime des titres-services pour les services et emplois de proximité. Rapport annuel, Bruxelles, Service public fédéral Emploi, Travail et Concertation Sociale, Direction générale Emploi et Marché du Travail.

Kuźma Elzbieta (2012) Emergence d'une communauté transnationale dans l'espace migratoire européen. Analyse de la migration polonaise à Bruxelles (2002-2009), Thèse défendue le 18 décembre, Université Libre de Bruxelles, Promoteur: Andrea Rea.

Lutz Helma (2008) Gender in the migratory process, Conference on Theories of Migration and Social Change, St. Ann's College, Woodstock Road Oxford, [online] last checked on 16/01/2013. URL: http://www.imi.ox.ac.uk/pdfs/helmalutz-gender-in-migratory-processes

Martiniello Marco et Rea Andrea (2011) Des flux migratoires aux carrières migratoires, SociologieS, Dossiers, Migrations, pluralisation, ethnicisation des sociétés contemporaines, [en ligne] consulté le 16/01/2013. URL : http://sociologies.revues.org/3694 
Martiniello Marco, Rea Andrea, Timmerman Christine et Wets Joan (Éds.) (2010) Nouvelles migrations et nouveaux migrants en Belgique, Gent, Academia Press, $339 \mathrm{p}$.

Mozère Liane (2002) Des domestiques philippines à Paris : un marché mondial de la domesticité ?, Revue Tiers Monde, XLIII (170), pp. 371-396.

OIM (2011) REAB, Return and Emigration of Asylum seekers Ex-Belgium, Annual Report, $152 \mathrm{p}$.

OIM (2009) Assessment of Brazilian Migration Patterns and Assisted Voluntary Return Programme from selected European Member States to Brazil, rapport de Recherche, septembre 2007-février 2009, 113 p.

Portes Alejandro, Guarzino Luis E. and Landolt Patricia (1999) The Study of Transnationalism: Pitfalls and Promise of an Emergent Research Field, Ethnic and Racial Studies, 22 (2), pp. 217-237.

Rosenfeld Martin, Gois Pedro, Lenz Annika, Reyntjens Pascal et Rea Andrea (2009) Immigration Brésilienne en Europe. Dimension Transnationale, Hommes \& Migrations, 1281, pp. 54-63.

Rosenfeld Martin, Marcelle Hélène et Rea Andrea (2010) Opportunités du marché de l'emploi et carrières migratoire, in Marco Martiniello, Andrea Rea, Christiane Timmerman et Johan Wets Éds., Nouvelles migrations et nouveaux migrants en Belgique, Gent, Academia Press, pp. 119-176.

Yépez Ysabel et Herrera Gioconda (Éds.) (2008) Nouvelles migrations latinoaméricaines en Europe : Bilans et défis, Louvain-la-Neuve/Barcelone, Presses universitaires de Louvain (UCL)/Publications I Edicions de la universitat de Barcelona, $306 \mathrm{p}$.

Van Meeteren Masja, Engbersen Godfried and Van San Marion (2009) Striving for a better position: Aspirations and the role of Cultural, Economic, and Social capital for Irregular Migrants in Belgium, International Migration Review, 43 (4), pp. 881-907.

Waldinger Roger (1994) The making of an immigrant niche, International Migration Review, 28 (1), pp. 3-30. 


\section{Any Freitas et Marie Godin}

\section{Carrières migratoires des femmes latino-américaines dans le secteur de la domesticité à Bruxelles}

L'article analyse les parcours migratoires des femmes migrantes venues d'Amérique latine (du Brésil et de l'Équateur particulièrement) et insérées dans le secteur de la domesticité informelle à Bruxelles. En s'appuyant sur le concept de " carrière migratoire ", qui offre un cadre d'analyse dynamique des trajectoires des migrantes, les auteures montrent que, derrière une (apparente) homogénéité du groupe, les femmes ont différents modes d'entrée sur le " marché du travail domestique " et qu'elles adoptent différentes stratégies afin d'améliorer (ou quitter) les positions qu'elles y occupent initialement. La mobilisation des réseaux sociaux, les transformations des politiques de séjour et les différentes conceptions de soi et " des possibles " peuvent entraîner des changements $\mathrm{d}^{\prime}$ " aspirations " et in fine, des " carrières migratoires " des femmes. En s'intéressant à leurs projets migratoires et à leur façon de mobiliser les différents types de capital (social, culturel, économique), les auteures s'écartent de la vision oppressante de la " chaîne du care globale ", accordant une place majeure au changement et aux bifurcations possibles. Loin de nier une marge de manœuvre restreinte en termes de mobilité sociale, l'article met en lumière certaines configurations particulières qui permettent aux femmes de saisir les opportunités qui s'offrent à elles dans cette "nouvelle " forme de division transnationale du travail.

\section{Migratory Careers of Latin-American Women in the Domestic Sector in Brussels}

This article analyzes the migratory trajectories of migrant women from Latin America (especially Brazil and Ecuador), working in the informal domestic sector in Brussels. Drawing on the concept of "migratory career", which offers a dynamic analysis of migrants' trajectories, this paper shows that, behind a (apparent) homogeneity, these women develop different strategies not only to find a place in this "domestic labor market", but also to improve (or exit) the positions they occupy initially. The mobilization of social networks, policy changes, and transformations in the conceptions of self and of "what is possible" may lead to changes in migrant women "aspirations", and eventually their "careers". The focus on their migratory projects, and the way these women mobilize their different types of capital (social, cultural, economic), allows us to escape the victimizing perspective of an oppressive "global care chain", while giving an important place to change and possible bifurcations. Far from denying their limited social mobility, this article highlights the specific configurations which may allow migrant women to take full advantage of the opportunities opened to them in this "new" form of transnational division of labor. 


\section{Carreras migratorias de las mujeres latinoamericanas en el sector de la domesticidad de Bruselas}

En este artículo se analizan los itinerarios migratorios de las mujeres migrantes de América Latina (especialmente Brasil y Ecuador) inseridas en el sector informal de la domesticidad en Bruselas. Utilizando el concepto de "carrera migratoria", que proporciona un análisis dinámico de las trayectorias de los migrantes, este artículo demuestra que, detrás de una homogeneidad (aparente), estas mujeres no sólo tienen diferentes modalidades de entrada en el «el mercado laboral de la domesticidad", sino también, que adoptan diferentes estrategias para mejorar las posiciones que inicialmente ocupaban - os simplemente quitárselas. La movilización de las redes sociales, las modificaciones de las políticas públicas, sus diferentes concepciones de sí mismas y de "los posibles" pueden llevar a transformaciones de las "aspiraciones» y, en última instancia, de las "carreras" migratorias de las mujeres migrantes. Focalizando nuestra atención en sus proyectos migratorios, y en cómo estas mujeres movilizan sus diferentes tipos de capital (social, cultural, económico), nuestro enfoque nos permite escapar a la tendencia victimista de los análisis de la "cadena global del care» (global care chain), dando un papel más importante a los cambios personales y posibles de bifurcaciones. Lejos de negar una flexibilidad limitada en términos de movilidad social, este artículo pone de relieve algunas características específicas que permiten a las mujeres a que aprovechen las oportunidades disponibles en esta "nueva» división transnacional del trabajo. 